\title{
Dynamic analysis of a five degree of freedom robotic arm using MATLAB-Simulink Simscape
}

\author{
Mihai Crenganis ${ }^{1, *}$, Alexandru Barsan $^{1}$, Melania Tera ${ }^{1}$, and Anca Chicea $^{1}$ \\ 1 "Lucian Blaga” University of Sibiu, Engineering Faculty, MIE Department, Sibiu, Romania
}

\begin{abstract}
In this paper, a dynamic analysis for a 5 degree of freedom (DOF) robotic arm with serial topology is presented. The dynamic model of the robot is based on importing a tri-dimensional CAD model of the robot into Simulink ${ }^{\circledR}-$ Simscape ${ }^{\mathrm{TM}}-$ Multibody ${ }^{\mathrm{TM}}$. The dynamic model of the robot in Simscape is a necessary and important step in development of the mechanical structure of the robot. The correct choice of the electric motors is made according to the resistant joint torques determined by running the dynamic analysis. One can import complete $\mathrm{CAD}$ assemblies, including all masses, inertias, joints, constraints, and tri-dimensional geometries, into the model block. The first step for executing a dynamic analysis is to resolve the Inverse Kinematics (IK) problem for the redundant robot. The proposed method for solving the inverse kinematic problem for this type of structure is based on a geometric approach and validated afterwards using SimScape Multibody. Solving the inverse kinematics problem is a mandatory step in the dynamic analysis of the robot, this is required to drive the robot on certain user-imposed trajectories. The dynamic model of the serial robot is necessary for the simulation of motion, analysis of the robot's structure and design of optimal control algorithms.
\end{abstract}

\section{Introduction}

The benefits of introducing robots into the industry include control and productivity management and a marked increase in product quality. Robots can work day and night without getting tired or reducing their performance. They consistently achieve substantial cost price reductions primarily by reducing the consumption of raw materials and due to the automatic processing. The use of robots also brings advantages to the competitive market. Through the rapid development of industry and computing technology, we can observe the evolution of robots to intelligent generations that give them the characteristic to "understand" the environment in which they work. The general structure of robots depends very much on the utility and purpose for which they are produced. Generally, robots are made of components similar to those of human beings, so they have an anthropomorphic structure. They are designed to reproduce the behaviour of humans and animals. This quality of nonhuman systems of having human or human-like qualities is called anthropomorphism. The aim of this paper is to design an anthropomorphic robotic arm with five degrees of mobility, comparable to that of human beings and to examine, study and simulate its dynamics and

* Corresponding author: mihai.crenganis@ulbsibiu.ro 
kinematics. The main goal of this paper is to create an anthropomorphic, redundant robotic arm with a simple structure, light weight, capable of manipulating objects with a mass of up to $0.2[\mathrm{Kg}]$ and a minimum energy consumption and which can be attached to a mobile platform with high manoeuvrability, such as the one in the below Figure 1.
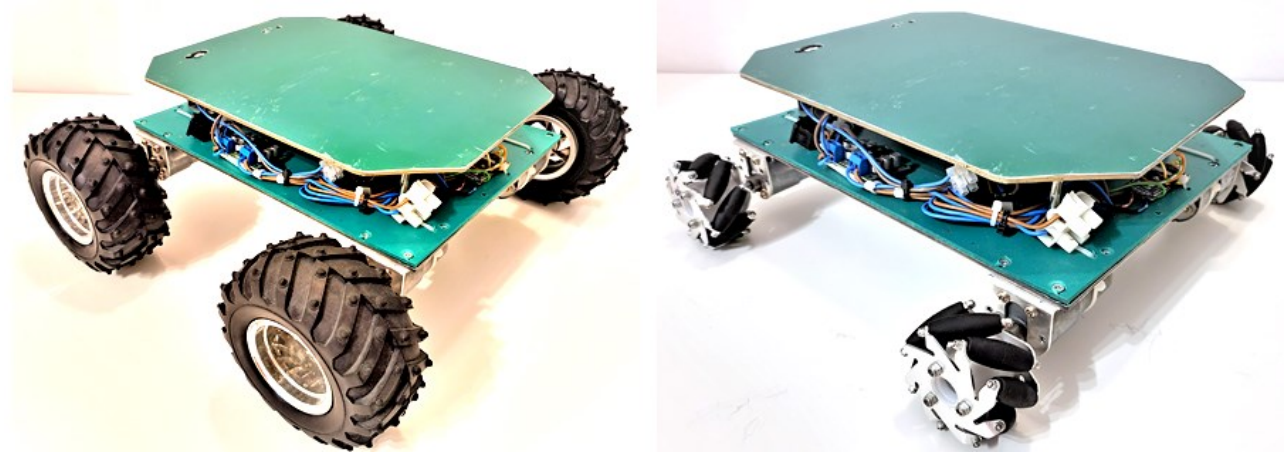

Fig. 1. Four-wheel drive platform for Mobile Manipulation

During the development of the project, aspects related to the mechanical structure of the arm, design, the kinematic part of the robot and the part of simulation and manipulation of a virtual model of the robotic arm were materialized. The conceptual result is a manipulator which acquires five degrees of mobility and a workspace comparable in shape to one of the human superior limbs, the dexterity being obtained with the help of the 5th class rotation joints. After consulting the literature, the mathematical equations for the corresponding kinematic part were determined, equations which are the foundation of a future virtual dynamic simulation [1-4].

\section{Kinematics of industrial manipulators and robots}

Kinematics deals with determining the main parameters that define the motion of a body. In this sense, various methods have been developed with which to determine the equations of positions, the distribution of speeds and the distribution of accelerations for any element in the structure of a kinematic chain [5-9]. Within the robot kinematics, two fundamental types of problems can be distinguished: direct kinematic problem and inverse kinematic problem. For the direct problem of positions, if the geometric characteristics of the kinematic chain and the laws of variation of the generalized coordinates are considered known, one must determine of the laws of variation of the absolute position and the absolute orientation of the characteristic point or the position of the final effector. Within the inverse kinematic problem, the geometric characteristics of the kinematic chain are considered known, as well as the laws of variation of the different absolute kinematic parameters and one must determine the relative kinematic parameters of the kinematic chain or the relative movements between two consecutive elements of the robot [10-13].

\subsection{Direct kinematics analysis of positions}

Knowing the relative parameters $\theta_{i}(i=1 . .5)$ and the shape of the homogeneous transfer matrices between elements, respectively between the coordinate systems attached to the elements, one can determine the total transfer matrix between the system $\mathrm{k}_{7}\left(\mathrm{x}_{7}, \mathrm{y}_{7}, \mathrm{z}_{7}\right)$ and the system $\mathrm{k}_{0}\left(\mathrm{x}_{0}, \mathrm{y}_{0}, \mathrm{z}_{0}\right)$. 


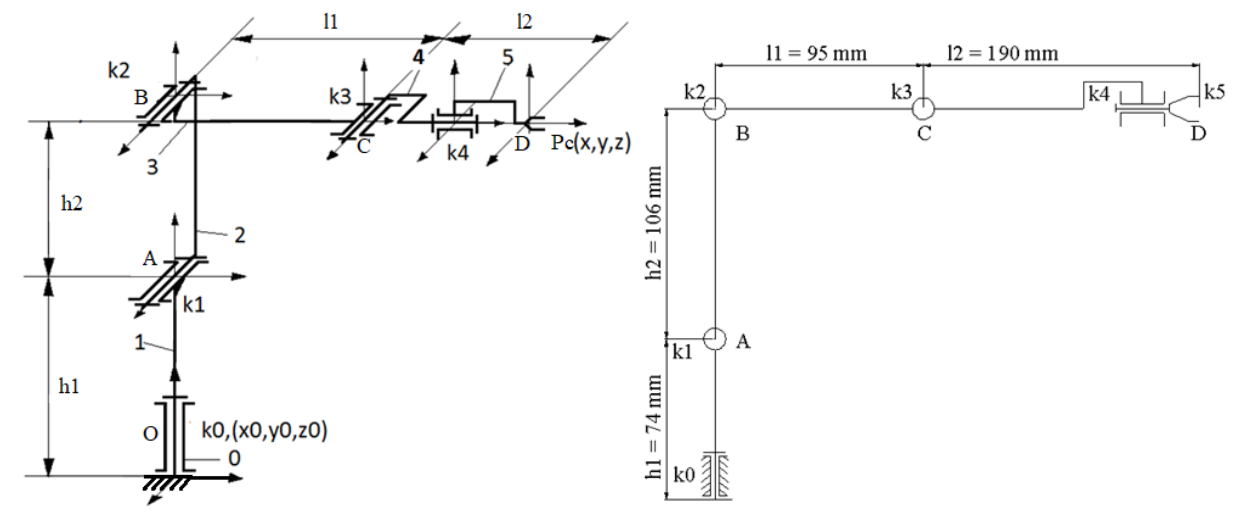

Fig. 2. Kinematic diagram of the 5 DOF robotic arm

Based on the above kinematic diagram of the robot, one can compute the direct and the inverse kinematics of the robotic arm. The first step in resolving the kinematics of the robot is to compute the total homogenous matrix that characterise the entire movement of the robot.

$$
\mathrm{H}_{05}=\mathrm{A}_{1} \cdot \mathrm{A}_{2} \cdot \mathrm{A}_{3} \cdot \mathrm{A}_{4} \cdot \mathrm{A}_{5} \cdot \mathrm{A}_{6} \cdot \mathrm{A}_{7} \cdot \mathrm{A}_{8} \cdot \mathrm{A}_{9}
$$

Were:

$\mathrm{A}_{1}=\mathrm{R}_{\mathrm{z}}\left(\theta_{l}\right) ; \mathrm{A}_{2}=\mathrm{T}_{\mathrm{z}}(h 1) ; \mathrm{A}_{3}=\mathrm{R}_{\mathrm{y}}\left(\theta_{2}\right) ; \mathrm{A}_{4}=\mathrm{T}_{\mathrm{z}}(h 2) ; \mathrm{A}_{5}=\mathrm{R}_{\mathrm{y}}\left(\theta_{3}\right) ; \mathrm{A}_{6}=\mathrm{T}_{\mathrm{x}}(l l) ; \mathrm{A}_{7}=$ $\mathrm{R}_{\mathrm{y}}\left(\theta_{4}\right) ; \mathrm{A}_{8}=\mathrm{R}_{\mathrm{x}}\left(\theta_{4}\right) ; \mathrm{A}_{9}=\mathrm{T}_{\mathrm{x}}(l 2)$.

The position and orientation of the end effector is expressed by the matrix:

$$
\mathrm{H}_{05}=\left[\begin{array}{llll}
a_{11} & a_{12} & a_{13} & a_{14} \\
a_{21} & a_{22} & a_{23} & a_{24} \\
a_{31} & a_{32} & a_{33} & a_{34} \\
a_{41} & a_{42} & a_{43} & a_{44}
\end{array}\right]
$$

Using the Euler angles or the Pitch, Roll and Yaw angles, it results that the general transfer matrix that characterizes the absolute orientation of the robot, respectively: rotation around the OX axis with angle $\varphi_{x}$, rotation around the OY axis with angle $\varphi_{y}$ and rotation around the $\mathrm{OZ}$ axis with angle $\varphi_{z}$ is of the form:

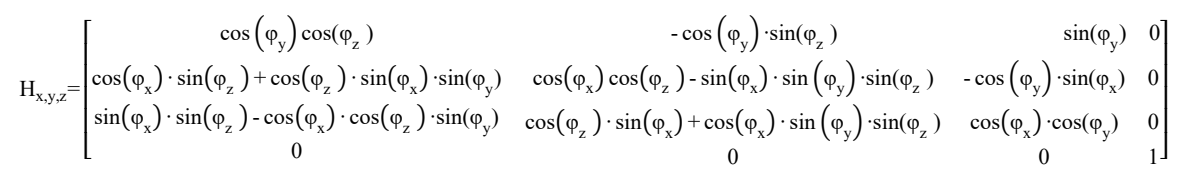

Equating the matrices (2) and (3) one obtains the following system of equations:

$$
\left\{\begin{array}{l}
\varphi_{\mathrm{x}}=-\arctan 2\left(a_{23}, a_{33}\right) \\
\varphi_{\mathrm{y}}=\arcsin \left(a_{13}\right) \\
\varphi_{\mathrm{z}}=-\arctan 2\left(a_{12}, a_{11}\right)
\end{array}\right.
$$

respectively the absolute position of the final effector:

$$
\left\{\begin{array}{l}
\mathrm{X}=a_{14} \\
\mathrm{Y}=a_{24} \\
\mathrm{Z}=a_{34}
\end{array}\right.
$$


In principle, this is the way to solve the direct kinematic problem for serial robots, by determining the homogeneous transfer matrices that characterize the structure of the robotic arm, followed by multiplying the matrices one by one, respecting the established order. Subsequently, the position and orientation of the end effector are extracted from the total transfer matrix $H_{i}$ using the expressions (4), (5).

\subsection{Inverse kinematic analysis of positions}

To solve this type of kinematic problem we start from the fact that we know the position and orientation of the final effector $\mathrm{x}, \mathrm{y}, \mathrm{z}, \varphi_{x} \varphi_{y}, \varphi_{z}$ with respect to a fixed reference system and we want to find the relative positions between the robot elements. In $3 \mathrm{D}$ space it is known that a body can have six degrees of mobility. Thus, if a coordinate system $k_{i} x_{i}, y_{i}, z_{i}$ is attached to this body, the position and orientation of this body with respect to a fixed coordinate system $k_{0}, O_{0}, x_{0}, y_{0}, z_{0}$ is expressed by the absolute parameters $x, y, z, \varphi_{x} \varphi_{y}$, $\varphi_{z}$ where:

$x$ - represents the projection on the axis $O_{0} \mathrm{x}_{0}$, of the distance from $k_{i}$ to $k_{0}$;

$y$ - represents the projection on the axis $O_{0} \mathrm{y}_{0}$, of the distance from $k_{i}$ to $k_{0}$;

$z$ - represents the projection on the axis $O_{0} \mathrm{z}_{0}$, of the distance from $k_{i}$ to $k_{0}$;

$\varphi_{x}$ - represents the rotation of the coordinate system $k_{i}$ around the axis $O_{0} x_{0}$;

$\varphi_{y}$ - represents the rotation of the coordinate system $k_{i}$ around the axis $O_{0} y_{0}$;

$\varphi_{z}$ - represents the rotation of the coordinate system $k_{i}$ around the axis $O_{0} z_{0}$;

As a result, it is desired to determine the relative parameters $\theta_{i}$ which represent the rotations between elements at the level of kinematic joints. In this case $(i=1 . .5)$;
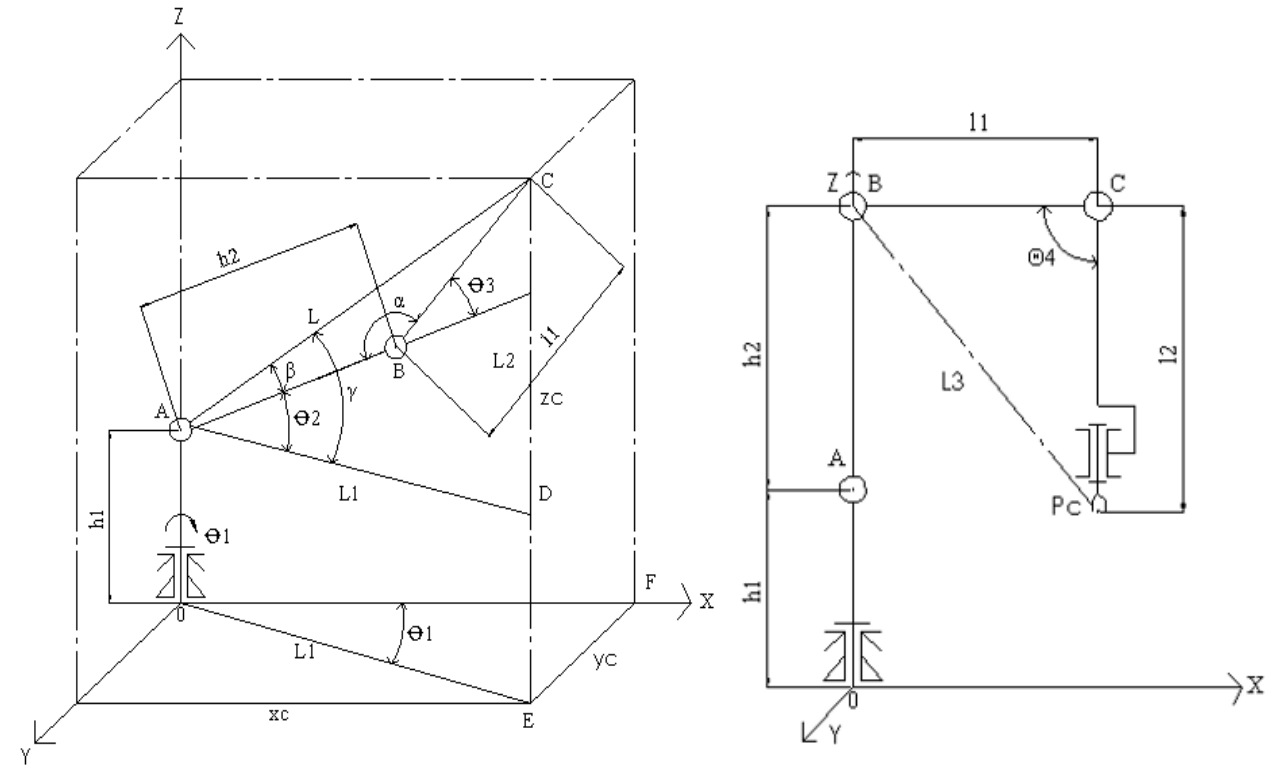

Fig. 3. Schematic used for indirect kinematics

Homogeneous transfer matrices are $A_{1}, A_{2}, A_{3}, A_{4}, A_{5}, A_{6}, A_{7}, A_{8}, A_{9}$. The relative movement from the first joint, $\theta_{1}$ is determined using the formula:

$$
\theta_{1}=\operatorname{atan} 2(y, x)
$$

To determine the position of $\mathrm{C}$ joint one can use the next system of equations: 


$$
H=A_{1} \cdot A_{2} \cdot A_{3} \cdot A_{4} \cdot A_{5} \cdot A_{6} \cdot A_{7} \cdot A_{8} \cdot A_{9}
$$

$H c=H \cdot A_{9}^{-1}$, from where it results:

$$
\left\{\begin{array} { l } 
{ x _ { C } = H c ( 1 , 4 ) } \\
{ y _ { C } = H c ( 2 , 4 ) } \\
{ z _ { C } = H c ( 3 , 4 ) }
\end{array} \quad \text { and } \quad \left\{\begin{array}{l}
x_{A}=0 \\
y_{A}=0 \\
z_{A}=h_{1}
\end{array}\right.\right.
$$

The distance from $\mathrm{C}$ joint to A joint can be determined using the following expression:

$$
L=\sqrt{\left(x_{C}-x_{A}\right)^{2}+\left(y_{C}-y_{A}\right)^{2}+\left(z_{C}-z_{A}\right)^{2}}
$$

For $\theta_{3}$ angle the generalized Pythagorean formula will be used, as following:

$$
\theta_{3}=\operatorname{acos}\left(\frac{L^{2}-h 2^{2}-l_{I}^{2}}{-2 \cdot h 2 \cdot l_{1}}\right)
$$

In the $\mathrm{BCD}$ triangle, using the same theorem (P.G.):

$$
l_{1}^{2}=L^{2}+h_{2}^{2}-2 \cdot L \cdot h_{2} \cdot \cos \beta
$$

Next one can determine $\beta$ angle:

$$
\beta=\arccos \left(\frac{l^{2}-L^{2}-h_{2}^{2}}{-2 \cdot L \cdot h}\right)
$$

In the ACD triangle, using the P.G theorem one can determine:

$$
L_{2}^{2}=L^{2}+h_{1}^{2}-2 \cdot L \cdot h_{1} \cdot \cos \alpha
$$

The $\alpha$ angle cand be determined using the expression:

$$
\alpha=\arccos \left(\frac{L_{2}^{2}-L^{2}-h_{1}^{2}}{-2 \cdot L \cdot h_{1}}\right)
$$

Knowing $\alpha$ and $\beta$ angles we calculate the value of $\theta_{2}$ :

$$
\theta_{2}=\alpha+\beta
$$

The following change, $B=A_{1} \cdot A_{2} \cdot A_{3} \cdot A_{4} \cdot A_{5} \cdot A_{6}$ is made in the total transfer matrix $H$, because they are known, and it results that:

$$
H=B \cdot A_{7} \cdot A_{8} \cdot A_{9}
$$

This means that:

$$
B=\pi \cdot A_{1-6}
$$

From the above system of equations (16) it results:

$$
\begin{gathered}
A_{7} \cdot A_{8}=B^{-1} \cdot H \cdot A_{9}^{-1} \\
{\left[\begin{array}{cccc}
\cos \left(\theta_{4}\right) & \mathrm{x} & \mathrm{x} & 0 \\
0 & \cos \left(\theta_{5}\right) & -\sin \left(\theta_{5}\right) & 0 \\
-\sin \left(\theta_{4}\right) & \mathrm{x} & \mathrm{x} & 0 \\
0 & 0 & 0 & 1
\end{array}\right]=\left[\begin{array}{cccc}
c_{11} & c_{12} & c_{13} & c_{14} \\
c_{21} & c_{22} & c_{23} & c_{24} \\
c_{31} & c_{32} & c_{33} & c_{34} \\
0 & 0 & 0 & 1
\end{array}\right],}
\end{gathered}
$$


Knowing the values for all $c_{i, j}$, it results that:

$$
\frac{-\sin \left(\theta_{4}\right)}{\cos \left(\theta_{4}\right)}=\frac{c_{31}}{c_{11}}
$$

and:

$$
\theta_{4}=-\operatorname{atan} \frac{c_{31}}{c_{11}}
$$

and:

$$
\frac{\sin \left(\theta_{5}\right)}{\cos \left(\theta_{5}\right)}=\frac{c_{23}}{c_{22}}
$$

From which we determine $\theta_{5}$ :

$$
\theta_{5}=-\operatorname{atan} \frac{c_{23}}{c_{22}}
$$

Therefore, the solutions to the inverse kinematics of the serial robot with 5 degrees of freedom, is:

$$
\left\{\begin{array}{c}
\theta_{1}=\operatorname{atan} \frac{y}{x} \\
\theta_{2}=\alpha+\beta \\
\theta_{3}=\operatorname{acos}\left(\frac{L^{2}-h^{2}-l_{1}^{2}}{-2 \cdot h \cdot l_{1}}\right) \\
\theta_{4}=-\operatorname{atan} \frac{c_{31}}{c_{11}} \\
\theta_{5}=-\operatorname{atan} \frac{c_{23}}{c_{22}}
\end{array}\right.
$$

\section{Robotic arm design}

This chapter includes aspects related to the development of the mechanical and the electronic part of this robot, but also aspects regarding the dynamic analysis of the robot. One of the main objectives of the research was to develop a small payload robotic arm with 5 DOF and reduced power consumption. The robotic arm must equip a four-wheel drive mobile robot. A mandatory criterion in the development of the robot was choosing the right electrical motors so that the robot could perform imposed tasks. Because the robotic arm is mounted on an autonomous vehicle the power drawn from the vehicle battery should be minimized. The dynamic analysis of the structure contributes to the right sizing of the electrical motors.

\subsection{Pre-determination of joint torques}

The next step is to pre-determine the joint torques for the robotic arm so one can model the robot intro a 3D CAD software. For this step, the following issues were considered:

- the whole ensemble was divided into three subassemblies.

- the maximum length of subassembly 1 is $\mathrm{h}_{2}=106[\mathrm{~mm}]$, of subassembly 2 is $\mathrm{l}_{1}=95$ $[\mathrm{mm}]$ and of subassembly 3 is $1_{2}=190[\mathrm{~mm}]$;

- the centres of gravity for subassemblies were considered to be placed in the middle of segments $\mathrm{h}_{2}, \mathrm{l}_{1}$ and $\mathrm{l}_{2}$;

- it was approximated that the maximum mass of the subassembly is $m_{1}=0.2[\mathrm{Kg}]$, of the subassembly 2 is $\mathrm{m}_{2}=0.2[\mathrm{Kg}]$ and of the subassembly 3 is $\mathrm{m}_{3}=0.2[\mathrm{Kg}]$;

- the mass of the manipulated part si $\mathrm{m}_{\mathrm{p}}=0.2[\mathrm{Kg}]$; 
- the total length of the robot is approximately $\mathrm{Lt}=391[\mathrm{~mm}]$;

- the mass of the whole assembly is $\mathrm{ms}=0.6[\mathrm{Kg}]$

Determination of the joint toques $T_{2}, T_{3}, T_{4}$ were performed for the following hypotheses, particular positions, presented in Figure 4.

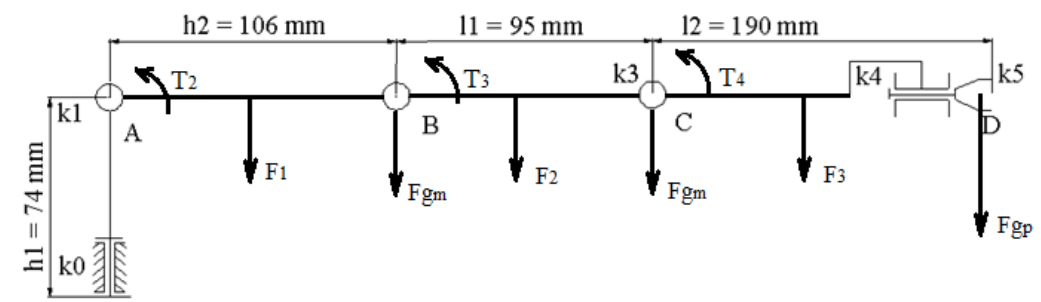

Fig. 4. Schematic used for pre-determination of joint torques

$$
\begin{gathered}
\mathrm{T} 2=-\left[m_{p} \cdot g \cdot\left(h_{2}+l_{1}+l_{2}\right)+m_{3} \cdot g \cdot\left(h_{2}+l_{1}+l_{2} / 2\right)+m_{2} \cdot g \cdot\left(h_{2}+l_{1} / 2\right)+m_{1} \cdot g \cdot\left(\mathrm{h}_{2} / 2\right)\right] \\
\mathrm{T} 2=-[0.2 \cdot 9.81 \cdot(0.106+0.095+0.19)+0.2 \cdot 9.81 \cdot(0.106+0.095+0.095)+ \\
0.2 \cdot 9.81 \cdot(0.106+0.0475)+0.2 \cdot 9.81 \cdot(0.053)]=-1.7[\mathrm{Nm}] \\
\mathrm{T} 3=-\left[m_{p} \cdot g \cdot\left(l_{1}+l_{2}\right)+m_{3} \cdot g \cdot\left(l_{1}+l_{2} / 2\right)+m_{2} \cdot g \cdot\left(l_{1} / 2\right)\right] \\
\mathrm{T} 3=-[0.2 \cdot 9.81 \cdot(0.095+0.19)+0.2 \cdot 9.81 \cdot(0.095+0.095)+ \\
0.2 \cdot 9.81 \cdot(0.0475)]=-0.93[\mathrm{Nm}] \\
\mathrm{T} 4=-\left[m_{p} \cdot g \cdot\left(l_{2}\right)+m_{3} \cdot g \cdot\left(l_{2} / 2\right)\right] \\
\mathrm{T} 4=-[0.2 \cdot 9.81 \cdot(0.095+0.19)+ \\
0.2 \cdot 9.81 \cdot(0.0475)]=-0.65[\mathrm{Nm}]
\end{gathered}
$$

\subsection{Choice of servomotors specific to each kinematic coupling}

The choice of the electrical servo motors was made based on the above results. Following the calculations, DC servo motors manufactured by Power HD were used to actuate the kinematic joints. The servomotor model used in robot structure is Power HD High Torque $1501 \mathrm{MG}$ for each joint. In the second kinematic joint two Power HD servo motors were used. It is one of the most popular servos with unique M/P technology and metal gear technology. This model offers one of the most powerful torque and speed available in any servo. The characteristics are: powerful three-pole ferrite motor, light weight metal gears, is equipped with a revolutionary metal / plastic case, has an output shaft with double ball bearing support.
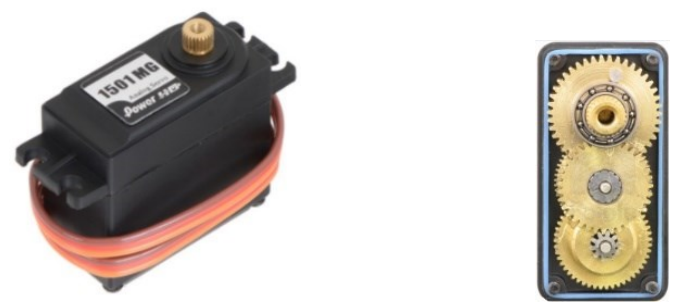

Fig. 5. Power HD High torque $1501 \mathrm{MG}$ servomotor

The general specifications of the servo motor are the following: size $40 \times 20 \times 40 \mathrm{~mm}$, weight $0.060[\mathrm{Kg}]$, operating principle - analogue type, angular velocity $7.5[\mathrm{rad} / \mathrm{s}]$, stall torque 1.7 [Nm]. 


\subsection{Mechanical structure and design of the robotic arm}

The mechanical structure of the robotic arm was designed using a 3D CAD program. After the 3D model was completed the virtual structure was converted into the Simulink-Simscape environment. Simscape offers to the users the possibility to analyse the dynamic behaviour of a kinematic chain in such way that one can introduce as input data, the forces acting on a mechanism and its desired motion and as output data one can collect the forces transmitted through the entire kinematic chain. The 3D virtual assembly consists of five subassemblies and a clamping device that will be presented and described in the following images in isometric view.
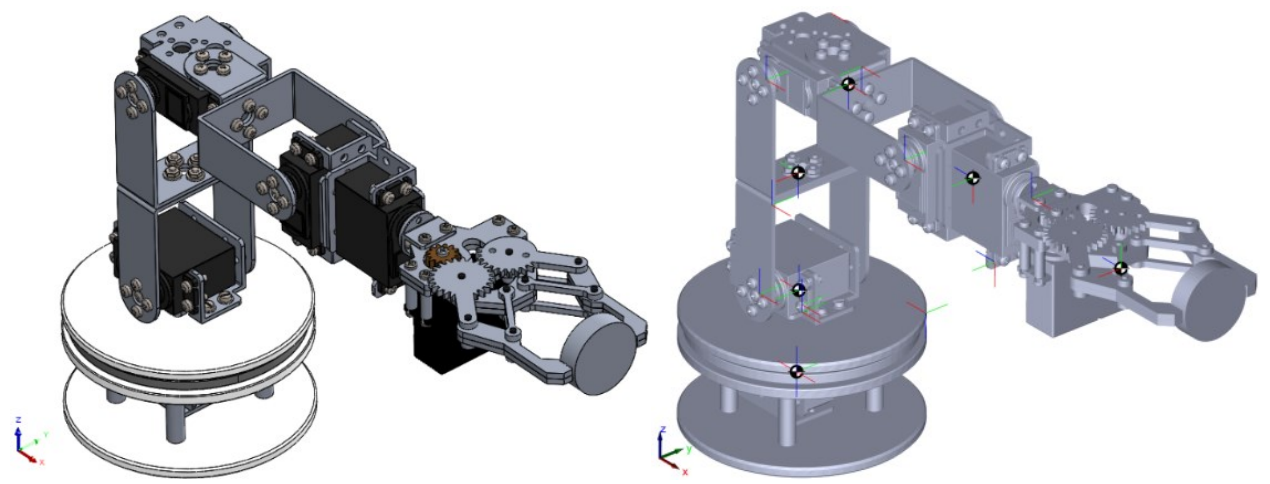

Fig. 6. Virtual 3D model of the 5DOF robotic arm CAD/Simulink-Simscape

\subsection{Dynamic analysis of the 5DOF robot}

The dynamic model of the 5 DOF robot is modelled in Simscape and is based on an import form a 3D CAD model. The Simscape virtual model is presented in Figure 7 and Figure 8.

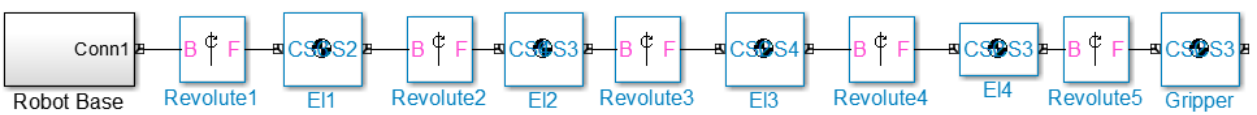

Fig. 7. Virtual Simscape model of the 5DOF robotic arm

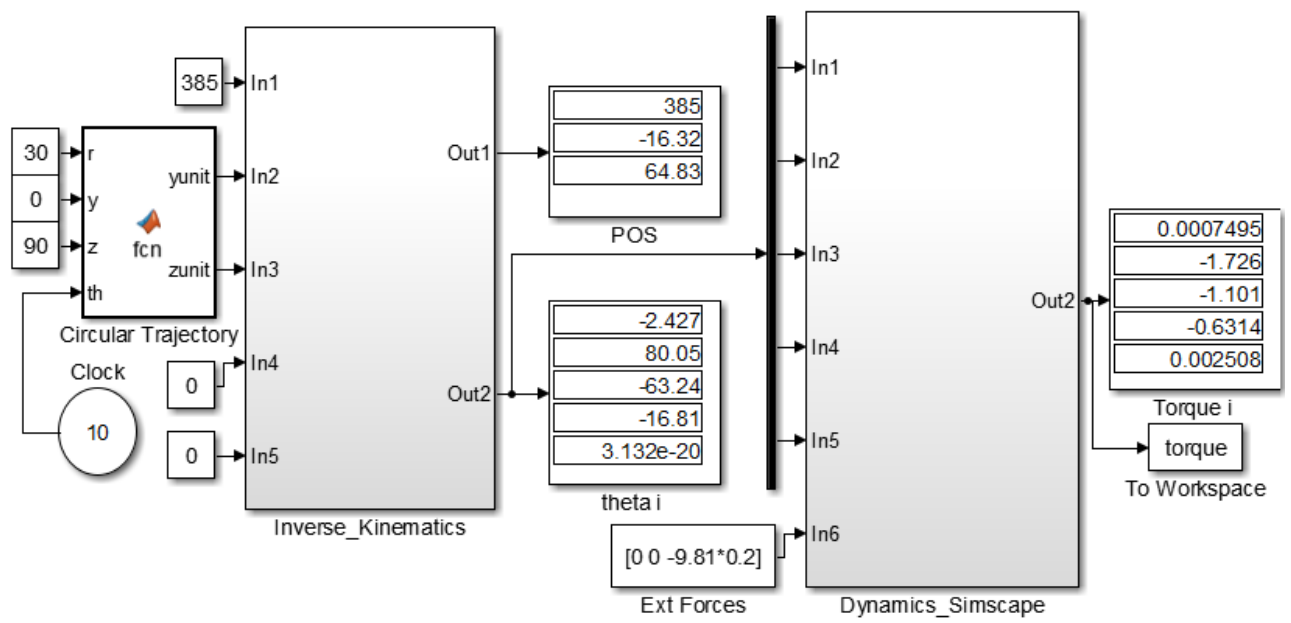

Fig. 8. Model block for dynamic analysis of the 5DOF robotic arm 
This virtual 3D model is necessary because all the robot physical characteristics like elements mass, inertia, dimension vector are imported in Simscape. The Simscape inverse dynamics model is based on kinematic equations of motion and by using differential equations one obtains the joint torques needed to produce the imposed motions. To obtain the joint torques necessary for a given trajectory we have imposed that the forces acting on the end effector are those resulted from manipulating a cylindrical body with the mass $\mathrm{m}=0.2[\mathrm{~kg}]$. So, the forces acting on the end effector during a circular trajectory are as follows: $\mathrm{Fz}=-9.81 \cdot 0.2$ $\mathrm{N} ; \mathrm{Fx}, \mathrm{Fy}=0 \mathrm{~N}$. As a result of end effector circular motion, the torques determined from the dynamic analysis have a sine wave variation. The torque variation is presented in Figures 9.
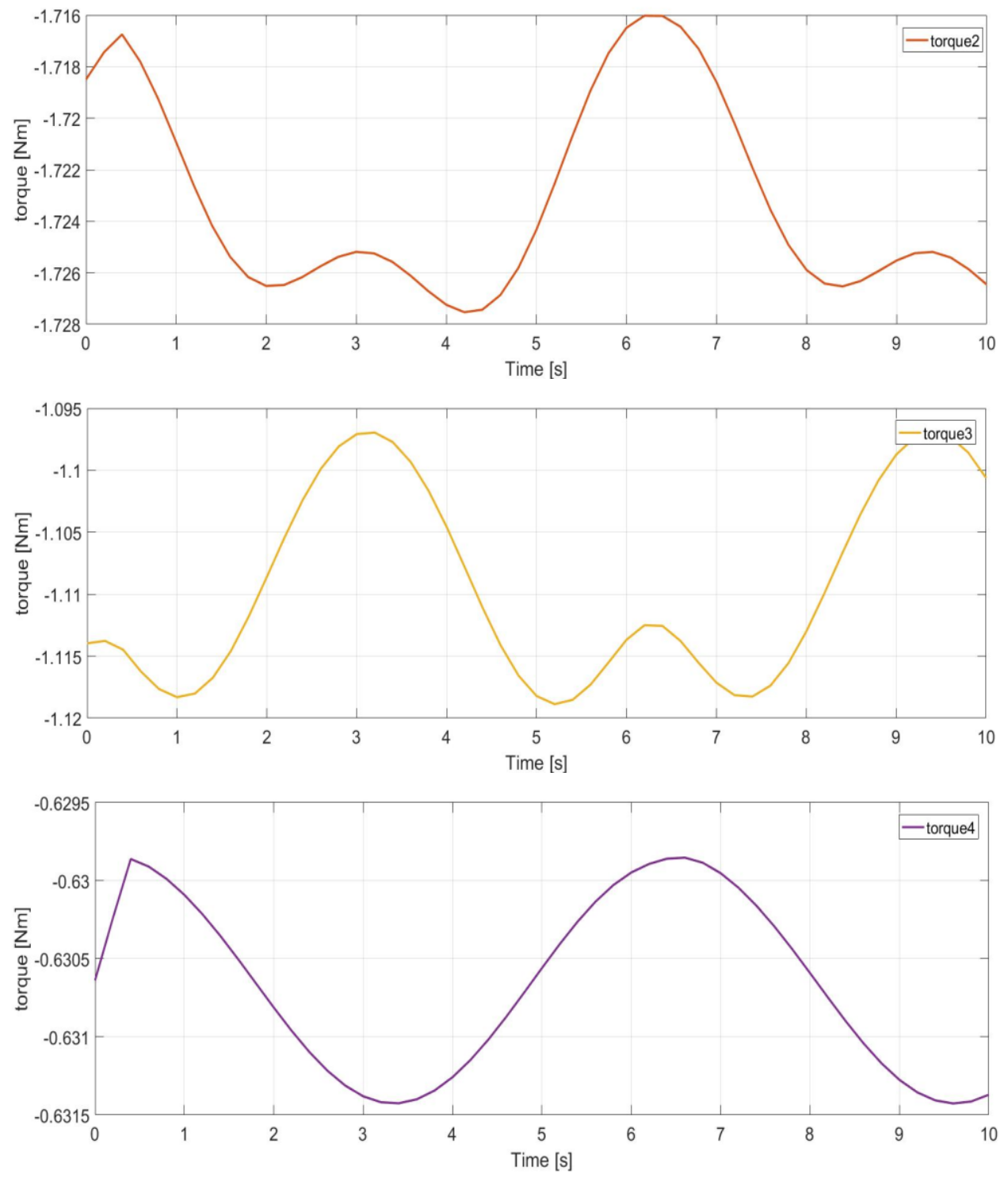

Fig. 9. Joint torques variation after running the dynamic analysis

After running the dynamic analysis one can observe that the maximum resistant joint torques registered are: $\mathrm{T} 1=0[\mathrm{Nm}], \mathrm{T} 2=-1.72[\mathrm{Nm}], \mathrm{T} 3=-1.101[\mathrm{Nm}], \mathrm{T} 4=-0.63[\mathrm{Nm}], \mathrm{T} 5=0$ $[\mathrm{Nm}]$. Because of these registered results the electrical servomotors were correctly chosen. 
The torque developed by Power HD High Torque 1501MG servomotor used in each kinematic joint is sufficient to pick and place a body with the mass of $0.2[\mathrm{Kg}]$. The physical structure of the 5 DOF robot developed after the entire dynamical analysis is presented in Figure 10 and Figure 11.

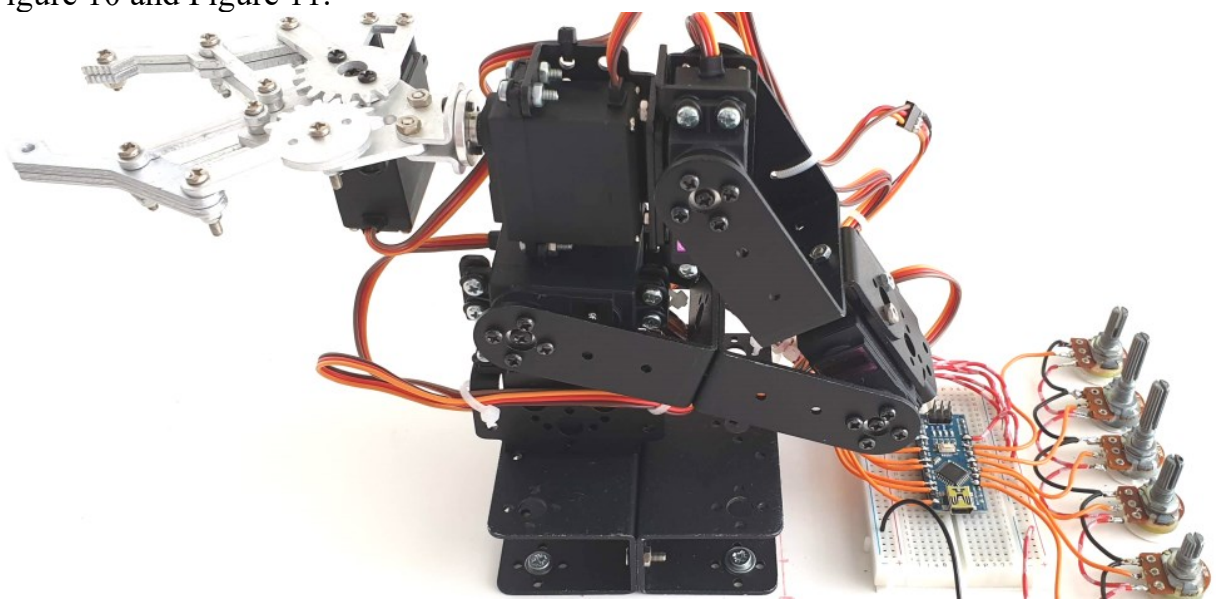

Fig. 10. 5DOF robotic arm
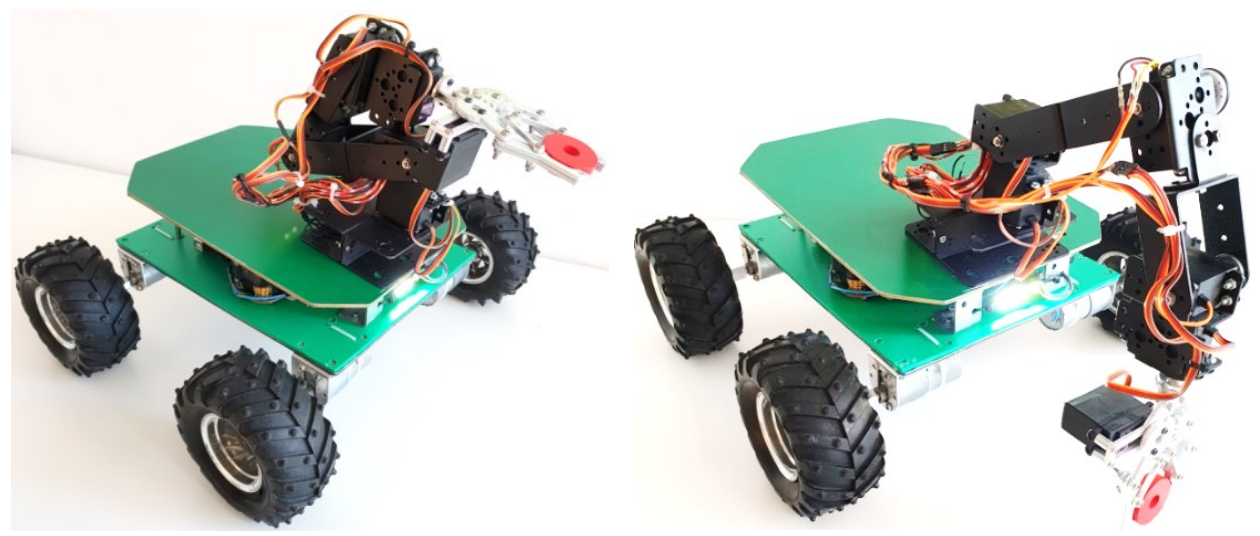

Fig. 11. 5DOF robotic arm mounted on a high manoeuvrability AGV

\section{Conclusions}

The main goal of this work was to design and develop an anthropomorphic robotic arm with five degrees of mobility with a simple structure, light weight and capable of manipulating objects with a mass of up to $0.2[\mathrm{Kg}]$ and a minimum energy consumption. An important aspect was that the robot can be attached to a mobile platform with high manoeuvrability. To be able to develop the robotic arm the kinematics and the dynamics of the robot had to be analysed. The kinematics was necessary to drive the robot arm through an imposed trajectory and the dynamic model was necessary to determine the joint resistant torques when the robot manipulates a body with the mass of $0.2[\mathrm{Kg}]$. The kinematics and the dynamics of the robot were simulated in Simulink-Simscape.

After simulations, the inverse kinematics equations and the dynamic model of the robot were correct and were validated during the virtual simulations. The two-analysis the static 
pre-dimensioning and the dynamic simulations share similar results, so the chosen PowerHD electrical servomotors are sufficient for manipulating some body elements with the maximum mass of $0.2[\mathrm{Kg}]$. The result of the research was a robot with a light weight and a total mass of $0.713[\mathrm{Kg}]$. From the PowerHD general specifications, the mechanical power of one servomotor is $\mathrm{Pm}=12.5 \mathrm{~W}$ so for the entire robot in the maximum extended position the total consumed power is $\operatorname{Pmmax}=25 \mathrm{~W}$, mechanical power and Pemax $=30 \mathrm{~W}$, electrical power.

\section{Acknowledgement}

This work was supported by a grant of the Romanian Ministry of Research and Innovation, CCCDI - UEFISCDI, project number PN-III-P1-1.2-PCCDI-2017-0446/82PCCDI/201, with in PNCDI III".

\section{References}

1. J. Angeles, Fundamentals of Robotic Mechanical Systems: Theory, Methods, and Algorithms, (Springer, 2006)

2. B. Siciliano, Handbook of Robotics, (Springer, 2016)

3. O. Bologa, R.E. Breaz, S.G. Racz, IJCCC, 10, 803-811, (2015)

4. G. Muresan, L. Morar, R.E. Breaz, Teaching CAM Techniques and CNC Programming in Technical Universities - An Integrated Approach, (Valencia, Spain, 2014)

5. S.G. Racz,R.E. Breaz, L.I. Cioca, Evaluating Safety Systems for Machine Tools with Computer Numerical Control using Analytic Hierarchy Process, SAFETY, 5, (2019)

6. A. Marosan, G Constantin, A Chicea, et. al., A Bârsan, Acta Universitatis Cibiniensis, 72, 49-56, (2020)

7. A. Marosan, G Constantin, A Barsan, et. al, Creating an ethernet communication between a Simatic S7-1200 PLC and Arduino Mega for an omnidirectional mobile platform and industrial equipment, IOP Conference Series: Materials Science and Engineering, (Galati, Romania, 2020)

8. M. Crenganis, M. Tera, C. Biris, C. Girjob, ITQM 2019, Dynamic Analysis of a 7 DOF Robot Using Fuzzy Logic for Inverse Kinematics Problem, (Granada, Spain 2019)

9. O. Bologa, RE. Breaz, SG. Racz, M. Crenganis, ITQM 2016, Decision-making tool for moving from 3-axes to 5-axes CNC machine-tool, (Asan, Korea,2016)

10. M. Crenganis, R. Breaz, G. Racz, O. Bologa, ICARCV 2012, Inverse Kinematics of a 7 DOF Manipulator Using Adaptive Neuro-Fuzzy Inference Systems, (Guangzhou, China, 2012)

11. A. Marosan, G Constantin, ICMAS 2020, PID Controller Based on A Gyroscope Sensor for an Omnidirectional Mobile Platform, (Bucuresti, Romania, 2020)

12. C. Gîrjob, O. Bologa, G. Racz, C. Biriş, Applied Mechanics and Materials 760, 391-396 (2015) 